\title{
Immunotherapy... a pursuit race to tomorrow's medicines
}

First draft submitted: 26 May 2017; Accepted for publication: 26 May 2017; Published online: 3 August 2017

Keywords: adaptive $\bullet$ antibody $\bullet$ checkpoint $\bullet$ immunology $\bullet$ immunotherapy

- inflammation $\bullet$ inhibitors $\bullet$ innate $\bullet$ oncology $\bullet$ vaccine

In 1900, during his Croonian Lecture "On Immunity with Special Reference to Cell Life" held at the Royal Society of London, Paul Ehrlich envisaged the importance of immunology, defining it as a rich source of treasures for biology and therapeutics [1]. More than one century later, immunotherapy is coming of age as treatment paradigm not only in the fields of autoimmune and inflammatory disorders [2-4], but also in oncology with recent successes of immune checkpoint inhibitors for treatment of different types of cancer [5-7]. Generally speaking, there are different categorical groups of immunotherapies including cell-based therapies, antibody therapies, peptide therapies and small molecule therapies. These categories can be further classified into immunosuppressive and immunoadjuvant therapies, depending on the kind of regulation they exert on the immune system. While in the first case they are aimed to thwart exacerbated self-immune responses, the goal of the other is to mobilize immune cells to seek and destroy foreign pathogens and/or cancer cells. Diverse areas of immunotherapy research are nowadays being pursued with immune-oncology that is rapidly growing in the arena of drug discovery as one of the most promising field of development for novel therapeutic opportunities in cancer disease. In the first editorial article of this collection, $\mathrm{Ta}$ and McNaughton illustrate the concept of immunotherapy, focusing on antibodies as immunotherapeutics and discussing antibody mimetics as viable strategy to overcome some issues related to production and manipulation of canonical antibodies [8].

Although biologic drugs represent the majority of immunotherapeutics currently in clinical development and in pharmaceutical market, small-molecule drugs targeting immunomodulatory pathways may provide advantages over antibodies, or can be even synergistic with biologic drugs. As discussed by Sasikumar and Ramachandra, developing small molecules for immune checkpoint antagonism may, for instance, lower the cost associated with drug manufacturing and drug administration of current approved immune checkpoint antibodies [9]. Small molecules are indeed more prone to oral bioavailability reaching greater exposure within tumor microenvironment, and eventually providing easy access therapies to patients with respect to biologic drugs. Notwithstanding, design and development of small molecules targeting immune checkpoint protein-protein interactions face inherent challenges, such as the identification and generally hydrophobic nature of transient pockets within the shallow interaction surfaces of protein binding partners that may increase cycle times of development and risk of late stage attrition in drug discovery pipeline. On the other hand, a greater knowledge on the complex immunoregulatory pathways in tumor microenvironment may offer alternative strategies, aiding the identification and validation of more conventional druggable targets, such as enzymes and receptors, that may enable faster and lower risk of attrition in lead compound to candidate progression. In this framework, enzymes of the first rate-limiting step of the kynurenine pathway, namely IDO1
Antonio Macchiarulo Department of Pharmaceutical Sciences, University of Perugia, Via del Liceo 1, 06123 Perugia, Italy antonio.macchiarulo@unipg.it newlands press part of 
and TDO2, have moved to the forefront of immunology as valuable drug targets for developing small molecule immunotherapies in oncology. In this regard, Jernigan and Sun discuss the relevance of IDO1 and TDO2 inhibition in the context of mechanisms of cancer immune escape, and provide an outlook on advanced computational approaches that may enable a fast and cost-effective identification of novel lead compounds to expand the chemical array of IDO1 and/or TDO2 inhibitors for clinical development [10]. Computational approaches may further combine with deployment of biophysical methods at early stage of drug discovery pipeline to aid selection of high-quality lead compounds, providing validation of target engagement and confirmation of functional activity. In our research article, we discuss the application of MicroScale Thermophoresis analysis to investigate binding properties of distinct categories of IDO1 inhibitors. Results pinpoint specific binding features of inhibitors, supporting the engagement of IDO1 by the clinical candidate indoximod as part of its still debated anticancer mechanism of action [11].

Design of host defense peptide (HPD) analogs is another strategy that holds promise for cancer immunotherapy. Specifically, HPD are cationic antimicrobial peptides produced by many living organisms, including mammals, as part of their innate immune system. Mostly studied as antibiotics for their antimicrobial activity, HPDs are recently gaining much interest for the selective cytotoxic activity they display against cancer cells. In their short communication, Sveinbjørnsson $\mathrm{et} \mathrm{al}$. discuss results of preclinical studies supporting the antitumor activity of a first in class oncolytic 9-mer peptide, namely LTX-315, that was designed on the basis of structure-activity relationships of the cationic antimicrobial peptide bovine lactoferricin [12]. LTX-315 is now advanced in Phase I clinical trials as monotherapy or in combination with immune checkpoint inhibitors for the treatment of different types of cancer.

While on the one side advancements in the field of immune-oncology herald breakthrough therapies for cancer patients, on the other they also reveal further barriers to tackle, which hamper full clinical responses to immunotherapeutic agents in some patients. Most of these barriers grounds on the lack of experimental immunotherapy models with a functioning immune system in preclinical studies. As discussed by Grohmann and collaborators, further efforts are required to boost the benefits from immunotherapy, especially those aimed at a better definition of clinically relevant screening platforms that may provide more reliable appraisals of the effectiveness of immunotherapeutic agents across a wide range of cancer types in preclinical testing [13].

In quest of next generation immunotherapies, some researchers are also looking at activating the innate immune system through small molecule agonists that target pattern recognition receptors (PRRs). In their review article, Mancini et al. elegantly discuss recent advances in the field of synthetic ligands of PRRs, including agonists of NOD-like receptors, RIG-I-like receptors and C-type lectin receptor [14]. Although these classes of PRRs are still poorly characterized, development of agonists can contribute to understand their immunoregulatory functions, paving the way to novel therapeutic opportunities from vaccine adjuvants to cancer immunotherapies. Jagadeesh Bayry further expands on this concept, commenting the use of PRRs agonists and chemokine receptor antagonists to transiently inhibit the activity of Tregs with the aim of boosting protective immunity to vaccines while keeping Tregs homeostatic functions in maintaining immune tolerance [15].

Targeting the interaction between selectins and cell adhesion molecule PSGL-1 with antibodies, glycopeptides or small molecules is a further emerging paradigm in immunotherapy. In particular, PSGL-1 plays a pivotal role in the early steps of leukocyte recruitment during inflammation. The commentary of Gerd Wagner highlights the key features of PSGL-1 and outlines different strategies for intervention with PSGL-1-mediated cell adhesion, focusing on the use of small molecules to downregulate PSGL-1 levels on leukocytes [16]. Finally, Kaur and Silakari discuss a totally different strategy that takes advantage of multitarget-oriented approaches [17]. Although they illustrate several examples of successful multitarget ligands from synthetic and natural sources in the context of inflammation, development of multitarget ligands may prove useful in the near future for the generation of innovative and more efficacious immunotherapeutic drugs for oncology.

\section{Conclusion}

Articles of this collection provide the reader with a glimpse on the vast array of potential therapeutic targets and related obstacles that the ever-increasing knowledge on immunoregulatory pathways is deploying for drug discovery. As a result, a pursuit race on part of both pharmaceutical companies and academic groups is getting to the heart for tomorrow's medicines.

\section{Financial \& competing interests disclosure}

A Macchiarulo is co-recipient with Professor Ursula Grohmann of a European Research Council grant (ERC-2013-AdG 338954-DIDO). The author has no other relevant affiliations or financial involvement with any organization or entity with a financial interest in or financial conflict with the subject matter or materials discussed in the manuscript apart from those disclosed.

No writing assistance was utilized in the production of this manuscript. 


\section{References}

1 Ehrlich P. On Immunity with Special Reference to Cell Life: Croonian Lecture. In: The Collected Papers of Paul Ehrlich, Vol. II: Immunology and Cancer Research. Himmelweit B (Ed.). Pergamon, London, UK, 148-192 (1956).

2 Feldmann M, Steinman L. Design of effective immunotherapy for human autoimmunity. Nature 435(7042), 612-619 (2005).

3 Hemmer B, Nessler S, Zhou D, Kieseier B, Hartung HP. Immunopathogenesis and immunotherapy of multiple sclerosis. Nat. Clin. Pract. Neurol. 2(4), 201-211 (2006).

4 Akdis CA. Therapies for allergic inflammation: refining strategies to induce tolerance. Nat. Med. 18(5), 736-749 (2012).

5 Pardoll DM. The blockade of immune checkpoints in cancer immunotherapy. Nat. Rev. Cancer 12(4), 252-264 (2012).

6 Mahoney KM, Rennert PD, Freeman GJ. Combination cancer immunotherapy and new immunomodulatory targets. Nat. Rev. Drug Discov. 14(8), 561-584 (2015).

7 Gotwals P, Cameron S, Cipolletta D et al. Prospects for combining targeted and conventional cancer therapy with immunotherapy. Nat. Rev. Cancer 17(5), 286-301 (2017).

8 Ta AN, McNaughton BR. Antibody and antibody mimetic immunotherapeutics. Future Med. Chem. 9(12), 1301-1304 (2017).

9 Sasikumar PG, Ramachandra M. Small molecule antagonists of the immune checkpoint pathways: concept to clinic. Future Med. Chem. 9(12), 1305-1308 (2017).
10 Jernigan FE, Sun L. In silico discovery and therapeutic potential of IDO1 and TDO2 inhibitors. Future Med. Chem. 9(12), 1309-1312 (2017).

11 Greco FA, Coletti A, Custodi C et al. Binding properties of different categories of IDO1 inhibitors: a microscale thermophoresis study. Future Med. Chem. 9(12), 1327-1338 (2017).

12 Sveinbjørnsson B, Camilio KA, Haug BE, Rekdal Ø. LTX-315: a first in class oncolytic peptide that reprograms the tumor microenvironment. Future Med. Chem. 9(12), 1339-1344 (2017).

13 Mondanelli G, Volpi C, Orabona C, Grohmann U. Challenges in the design of reliable immuno-oncology mouse models to inform drug development. Future Med. Chem. 9(12), 1313-1318 (2017).

14 Neilsen AE, Hantho JD, Mancini RJ. Synthetic agonists of NOD-like, RIG-I-like, and C-type lectin receptors for probing the inflammatory immune response. Future Med. Chem. 9(12), 1345-1360 (2017).

15 Bayry J. Harnessing the regulators to enhance viral vaccine efficacy. Future Med. Chem. 9(12), 1319-1322 (2017).

16 Wagner G. Down regulation of P selectin glycoproteins as a potential anti-inflammatory mechanism. Future Med. Chem. 9(12), 1323-1226 (2017).

17 Kaur G, Silakari O. Multiple-target centric strategy to tame Inflammation. Future Med. Chem. 9(12), 1361-1378 (2017). 